\title{
Conjugation Of EGCG And Chitosan NPs As A Novel Nano-Drug Delivery System
}

This article was published in the following Dove Press journal:

International Journal of Nanomedicine

\author{
Abdel-Majeed Safer (D) \\ Stefano Leporatti ${ }^{2}$ \\ Jacquilion Jose ${ }^{3}$ \\ Mahmoud S Soliman (1D ${ }^{4}$ \\ 'Department of Biological Sciences, \\ Faculty of Science, Kuwait University, \\ Kuwait City, Kuwait; ${ }^{2}$ CNR Nanotec, \\ Istituto di Nanotecnologia, Lecce 73/00, \\ Italy; ${ }^{3}$ Nanoscopy Science Center, Faculty \\ of Science, Kuwait University, Kuwait \\ City, Kuwait; ${ }^{4}$ Nanotechnology Research \\ Facility, Faculty of Engineering and \\ Petroleum, College of Engineering and \\ Petroleum, Kuwait University, Kuwait \\ City, Kuwait
}

Purpose: Chitosan nanoparticles (CS NPs) have been used as a good vehicle for nano-drug delivery due to their good physicochemical properties. Epigallocatechin-3-gallate (EGCG), one of the major active ingredients of green tea, is a natural antioxidant that helps in reducing and preventing cell damage and fighting cancer, plus providing other benefits. The aim of this study is to optimise the preparation parameters in terms of the physical characteristics and stability in CS/EGCG NPs conjugation.

Results: The conjugation of CS/EGCGNPs was obtained by means of Poloxamer 188. The average CS/EGCG NPs complex was of size $117.8 \pm 38.71 \mathrm{~nm}$ with a surface charge of +67.8 $\pm 4.38 \mathrm{mV}$ and isoelectric point at $\mathrm{pH} 7.61$.

Conclusion: In conclusion, NPs produced were stable at $4{ }^{\circ} \mathrm{C}$ with nanometric size, good polydispersity, good loading and efficiency, envisaging to be a possible candidate for nanotherapeutic delivery system against hepatic fibrosis.

Keywords: chitosan, EGCG, conjugation, nano-drug delivery

\section{Introduction}

Nano-drug delivery has attracted much attention due to its unique physical, chemical and biological properties. It has become a bright spot in biochemical research. It is emerging as a prospective biomedical tool with applications in diagnostics, specific drug delivery and therapeutics for diseases. That is to say, it is a method of delivering drugs to a biological system in a manner that increases the amount of the drug in such parts of the system with high affinity. In this technique, conjugated nanoparticles (NPs) and their carriers are employed. This mean of delivery is largely useful in making such drug reaching its target cells and tissues of an organ thereby smarter than the conventional manner. ${ }^{1}$ One of the major advantages of nano-drug delivery technique is to spontaneously allow the NPs to reach its specific diseased target, thereby avoiding interaction with healthy tissue. ${ }^{2}$ The goal of a drug delivery system is known to prolong the effects and to have a protected drug interaction with the diseased tissue, as the system releases the drug in a dosage form. ${ }^{3,4}$ The advantages of such system are the reduction in the frequency of the dosages taken by the patient, having a more uniform effect of the drug side effects, ${ }^{5}$ and reduced fluctuation in circulating drug levels, thereby preventing any damage of the healthy tissue, ${ }^{6}$ whereas the conventional delivery system is based on the absorption of the drug across a biological membrane, which depends on the type of the cell and its location in the body. ${ }^{7}$ However, the disadvantages of nano-drug delivery system still need to be tested.
Correspondence: Abdel-Majeed Safe Department of Biological Sciences, Faculty of Science, Kuwait University, Kuwait City, Kuwait

Tel +96524985909

Email saferam52@gmail.com

Stefano Leporatti

CNR Nanotec, Istituto di

Nanotecnologia, Lecce, Italy

Tel +390832319829

Email stefano.leporatti@nanotec.cnr.it 
Although gold NPs are not the main objective in this study, yet, the use of gold, chitosan, and epigallocatechin gallate (EGCG) NPs has recently been developed as a smart mean of drug delivery system for the treatment of various kinds of diseases, such as cancer, diabetes and neurodegenerative diseases. ${ }^{7,8}$ Drug delivery system is a promising technique in years to come, where it will overcome the conventional treatment for cancer, diabetes, gastro-intestinal disorder, endocrine diseases, etc. ${ }^{6-10}$ The aim of this work is to elaborate more on the use of such technique for a more efficient and better release of nanodrug for therapeutic treatment of hepatic fibrosis as one of the major diseases worldwide.

\section{Materials And Methods}

\section{Sample Preparation}

In the preparation of NPs, cleaning of glassware is very crucial. Thus, all the glassware and stirring magnetic bars were thoroughly cleaned in freshly prepared aqua regia $(\mathrm{HCl} / \mathrm{HNO} 33: 1, \mathrm{v} / \mathrm{v})$ and then rinsed with distilled water and dried, to avoid aggregation of residual gold particle and to avoid unwanted nucleation during synthesis.

\section{Preparation Of Gold NPs}

In this report, gold NPs have been prepared and used for the sake of morphological comparison with other NPs under study.

Ten milligrams of $\mathrm{HAuCl}_{4}$ was dissolved in $100 \mathrm{~mL}$ of deionized water $(0.25 \mathrm{mM})$, and shaken properly to $\mathrm{mix}$ the solution. Solution of reducing agent $\left(\mathrm{NaBH}_{4}\right)$ was prepared by dissolving 1.891 of $\mathrm{NaBH}_{4}$ in $500 \mathrm{~mL}$ of deionized water. Then, $100 \mathrm{~mL}$ of $\mathrm{HAuCl}_{4}(0.25 \mathrm{mM})$ was taken in $250 \mathrm{~mL}$ flask with magnetic stirring at 750 rpm and 50 drops of the reducing agent solution was added drop by drop with continuous stirring. The color of $\mathrm{HAuCl}_{4}$ solution changed to dark red over several seconds. Stirring process was continued for another 10 mins for complete homogenization. Since the $\mathrm{HAuCl}_{4}$ is corrosive, a glass spatula was used to avoid the contact with metal. All the gold NPs batches were stored in the dark to minimize the photo-induced oxidation. The UV absorption spectra were recorded between 190 and $1100 \mathrm{~nm}$ on spectrophotometer. The zeta potential was also measured. Particles size analysis was done using transmission electron microscope (TEM), scanning electron microscopy (SEM) and atomic force microscopy (AFM) images.

\section{Preparation Of Chitosan/TPP NPs}

Two percent acetic acid solution was prepared in deionized water. LMW chitosan was dissolved in the aqueous solution of acetic acid to form a $0.5 \mathrm{mg} / \mathrm{mL}$ chitosan solution. (Total $30 \mathrm{~mL}$ prepared, so $15 \mathrm{mg}$ of chitosan was dissolved in $30 \mathrm{~mL}$ of $2 \%$ acetic acid). The chitosan solution was stirred overnight at room temperature using a magnet stirrer. The $\mathrm{pH}$ of the resulting solution was around 3.6 and this was adjusted to 4.7-4.8 using $20 \mathrm{wt} \%$ aqueous sodium hydroxide solution. The chitosan solution was then passed through a syringe filter (pore size $0.45 \mu \mathrm{m}$, Millipore, USA) to remove residues of insoluble particles. Tripolyphosphate (TPP) was dissolved in ultrapure water at a concentration of $0.5 \mathrm{mg} / \mathrm{mL}$ and also passed through a syringe filter (pore size $0.45 \mu \mathrm{m}$, Millipore, USA). Ten milliliters of chitosan solution was taken in a vial and was then placed on the magnetic stirrer stirring at 700 rpm. $1.0 \mathrm{~mL}$ of $2-4^{\circ} \mathrm{C}$ TPP solution was quickly added to the chitosan solution with a micropipette. The reaction was carried out for 10 mins and the resulting suspension was subjected to further analysis. UV spectra scanned between 190 and 1000nm.

\section{Preparation Of Gold/Chitosan NPs}

A total of $100 \mu \mathrm{L}$ of $0.125 \mathrm{M}$ concentrated aqueous solution of chloroauric acid $\left(\mathrm{HAuCl}_{4}\right)$ was reduced by heating at $100^{\circ} \mathrm{C}$ for 15 mins in $100 \mathrm{~mL}$ of low molecular weight chitosan solution prepared in $1 \%$ acetic acid to yield a ruby-red solution. The ruby-red colored solution yielded an absorbance maximum at $520 \mathrm{~nm}$. Varying chitosan concentrations $(0.05 \%, 0.01 \%, 0.1 \%, 0.2 \%, 0.5 \%$ and $1 \%)$ were used for reduction of chloroauric acid. ${ }^{11}$

\section{Preparation Of EGCG/Chitosan NPs}

$0.1 \%$ Low molecular weight chitosan (Sigma) solution was prepared in $1 \%$ acetic acid. Twenty-five milligrams of Poloxamer $188^{12}$ was dissolved in $5 \mathrm{~mL}$ of the above chitosan solution. $0.05 \%$ EGCG (Sigma) solution was prepared in distilled water. $0.025 \%$ TPP solution (Merck) was prepared in distilled water. Five milliliters of chitosan solution was added to $50 \mathrm{~mL}$ of EGCG solution upon stirring at room temperature. To the above solution, $0.5 \mathrm{~mL}$ of TPP solution was added drop-wise under stirring for $3 \mathrm{hrs}$ at a speed of $1000 \mathrm{rpm}$. The solution formed was translucent. The solution was centrifuged at $14,000 \mathrm{rpm}$ for 10 mins using high-speed tabletop centrifuge (Hermle, Z36HK). The UV absorption spectra were recorded between 190 and $1100 \mathrm{~nm}$ on spectrophotometer (Cary 
win $50 \mathrm{UV})$. The particle size and zeta potential and isoelectric points were measured using Malvern Instrument (Nano ZS, green badge). Particle characteristics investigation and acquiring were done on a Jeol 1200 EXII electron microscope (JEOL Ltd, Tokyo, Japan), operated at $80 \mathrm{kV}$ with magnification from 150 to $200 \mathrm{~K}$, and on the variable pressure field emission remotely operated scanning electron microscope 9 Leo Supra $50 \mathrm{VP}$ operated at $30 \mathrm{kV}$ with magnification of $40 \mathrm{~K}$ and the Atomic Force Microscope Veeco Nanoscope IV Multimode AFM Model No. NMAFMLN (Figure 6) for fabrication of Poloxamer (P188) loaded chitosan NPs via ionic gelation between the positively charged amino groups of chitosan (CS) and negatively charged triphosphate (TPP) with EGCG trapped in.

\section{Transmission Electron Microscopy Preparation Of Gold NPs, Chitosan NPs, Gold/Chitosan NPs And EGCG/Chitosan NPs For TEM}

NPs preparations of gold, chitosan, gold/chitosan conjugation and EGCG/chitosan conjugation were done almost the same for examination in the TEM. Pasteur capillary pipette $150 \mathrm{~mm}$, rubber bulb, Formvar carbon-coated copper grids 150 mesh, tweezers for handling the grids and ultrasonicator were used during sample preparation for the TEM. The sample in the form of solution was ultrasonicated for 15 to 45 mins to insure homogeneity. Using the tweezers it was picked out the grid from the grid box and placed it on a filter paper previously set into a petri dish. $150 \mathrm{~mm}$ Pasteur glass pipette was used to take $1 \mathrm{~mL}$ of the desired specimen to be examined. The pipette was inserted into the solution just under the surface level at the center of the vial. One to 2 drops of solution was added on the Formvar carbon-coated grid and let dry in the desiccator to keep it safe from contamination. Thus, samples are ready for TEM examination. The grid was placed in the specimen holder using the tweezers for examination in STEM JEM-1200 EX II JEOL Scanning Transmission Electron Microscope at magnifications of 200 to $250 \mathrm{~K}$ operated at $80 \mathrm{kV}$.

\section{Field Emission Scanning Electron Microscopy (FESEM)}

Preparation Of Gold NPs, Chitosan NPs, Gold/Chitosan NPs And EGCG/Chitosan NPs For The FESEM

NPs preparations of gold, chitosan, gold/chitosan and EGCG/chitosan conjugation were done almost in the same way, for examination in the FESEM samples were prepared in the same way for the four NPs. The NPs were poured on washed clean cover slip and fixed on big stub ready for dehydration. The mounted materials were covered and left at room temperature for a few hours and later in a desiccator. The stubs were put in the critical point drying machine then in the sputter coater for shadowing with platinum/gold. Investigation and image acquisition were done on the variable pressure field emission remotely operable scanning electron microscope (Leo Supra 50VP) operated at $30 \mathrm{kV}$.

\section{ImageJ Analysis}

ImageJ software program (Freeware ImageJ V $1.51 \mathrm{~A}$ ) public domain image processing program developed at the National Institutes of Health ${ }^{13-15}$ was applied to images. Statistical analysis was performed: Gaussian size distribution (standard deviation), shape and error analysis of NPs were carried out using freeware ImageJ and statistical software Origin Pro2019 to plot the data excel sheet for UV and ZP and ZS.

\section{Atomic Force Microscopy (AFM)}

Preparation Of Gold NPs, Chitosan NPs, Gold/Chitosan NPs And EGCG/Chitosan NPs For The AFM

NPs solutions of gold, chitosan, gold/chitosan and EGCG/ chitosan were spread on a mica substrate on the AFM Stub and were allowed to dry overnight. The dried sample was then mounted on the AFM Multimode for scanning with TappingMode Tip. The AFM Multimode used was VEECO Nanoscope IV Multimode microscope and the Tapping Mode Tip used was RTESP tip. Topographical images (height mode) were acquired. Three-dimensional (3D) analysis was employed to distinguish roughness and morphological features.

\section{Fourier-Transform Infrared Spectroscopy (FTIR)}

The samples of the three solutions (EGCG, chitosan and EGCG/chitosan) were stored in vials in a freezer prior to freeze-drying at $-18^{\circ} \mathrm{C}$. The freeze dryer temperature and pressure were approximately $-40^{\circ} \mathrm{C}$ and $133 \times 10^{-3} \mathrm{mbar}$, respectively. The vials were then transferred into the lyophilisation flask and loaded onto the Freeze Dryer (Labconco, Freezone 18 USA). Once loaded, waited for 5 mins to verify that the pressure is $133 \times 10^{-3}$ mbar or lower. The flask was disconnected from the manifold one by one after the sample is completely dried and used for the FTIR analysis. The powder sample and $\mathrm{KBr}$ (Sigma) was ground to reduce the particle size to $<5 \mathrm{~mm}$ in diameter. $0.2 \mathrm{mg}$ of $\mathrm{KBr}$ was added into an agate mortar and 
ground it to fine powder until crystallites can no longer be seen, and it becomes somewhat "pasty" and sticks to the mortar. $2 \mathrm{mg}$ of powder sample was mixed with the $\mathrm{KBr}$ powder. The mixture was ground for 3-5 mins then it was put into the Pike-Press Pellet (Vacuum crusher, Model pike crush IR Pike Tech, Madison). The powder was then pressed for 2 mins to form a thin and transparent pellet. The pellet was taken out carefully and transferred onto the sample holder of the FTIR (Varian 640-IR spectrometer, Randburg) for analysis.

\section{X-Ray Photoelectron Spectroscopy (XPS) X-Ray Photoelectron Spectroscopy (ESCA Lab 250xi (Thermo))}

XPS spectra were recorded on a Thermo ESCALAB 250 $\mathrm{Xi}$ using monochromatic $\mathrm{Al} \mathrm{K} \alpha$ radiation $(1486.6 \mathrm{eV})$ with a spot size of $850 \mu \mathrm{m}$. The spectra acquisition and processing were carried out using the software Thermo Avantage. The sample was stuck on the sample holder using a double-sided carbon tape and then introduced into the preparation chamber and was degassed until the proper vacuum was achieved. Then, it was transferred into the analysis chamber where the vacuum was $10^{-9}$ mbar. The analysis was carried out using the following parameters: pass energy of $20 \mathrm{eV}$, dwell time $50 \mathrm{~ms}$ and the step size $0.1 \mathrm{eV}$.

\section{Results}

\section{Characterization} UV-Vis Spectroscopy

The UV-Vis absorption spectra of all samples were measured in the range $200-1000 \mathrm{~nm}$. The results showed that they have no absorption peaks in visible region for all samples. However, the maximum absorption peak for gold NPs was clearly detected at $520 \mathrm{~nm}$ (see Figure 1), whereas there was no absorption for chitosan, gold/chitosan and EGCG/chitosan.

\section{Zeta Potential And Size Measurements Of NPs}

According to the protocol used in this work, four different NPs have been used, namely gold NPs, in the form of $\mathrm{HAuCl} 4$ and reducing agent $(\mathrm{NaBH} 4)$. The NPs obtained were of size $47.03 \pm 45.55$ (d.nm), and PdI of 0.328 , zeta potential was $56 \mathrm{mV}$, as shown in Figure 2A. In comparison to the previous case chitosan TPP NPs of size 156 $\pm 45.55 \mathrm{~nm}$ and PdI 0.38, zeta potential $-12.9 \pm 8.07 \mathrm{mV}$ (Figure 2B). Gold/chitosan NPs have size of $160.3 \pm 36.17$ $\mathrm{nm}$ and have PdI of 0.581 whereas the zeta potential was of $63.9 \pm 4.01 \mathrm{mV}$ (as shown in Figure 2C). EGCG/chitosan NPs have size of $414.8 \pm 333.8 \mathrm{~nm}$ and PdI 0.363 , zeta potential of $67.6 \pm 4.38 \mathrm{mV}$ (Figure 2D). The size of the four NPs prepared in this work varied from small $(<200$ $\mathrm{nm})$ to large (more than $400 \mathrm{~nm}$ ). Isoelectric point for EGCG/chitosan was at a neutral point $\mathrm{pH} 7.4$ (see Figure 2E).

\section{Isoelectric Point}

The isoelectric points of the three components were calculated: EGCG has isoelectric point at pH 4.28 (EGCG), after centrifugation and auto-titration (reported in Figure 2F), chitosan/poloxamer weighted mean zeta potential (chitosan/poloxamer) has isoelectric point at $\mathrm{pH} 10.1$ after centrifugation (see Figure $2 \mathrm{G}$ ) and auto-titration. While for chitosan/EGCG conjugate, isoelectric point is at $\mathrm{pH} 6.89$ (chitosan/EGCG conjugation), after centrifugation and autotitration.

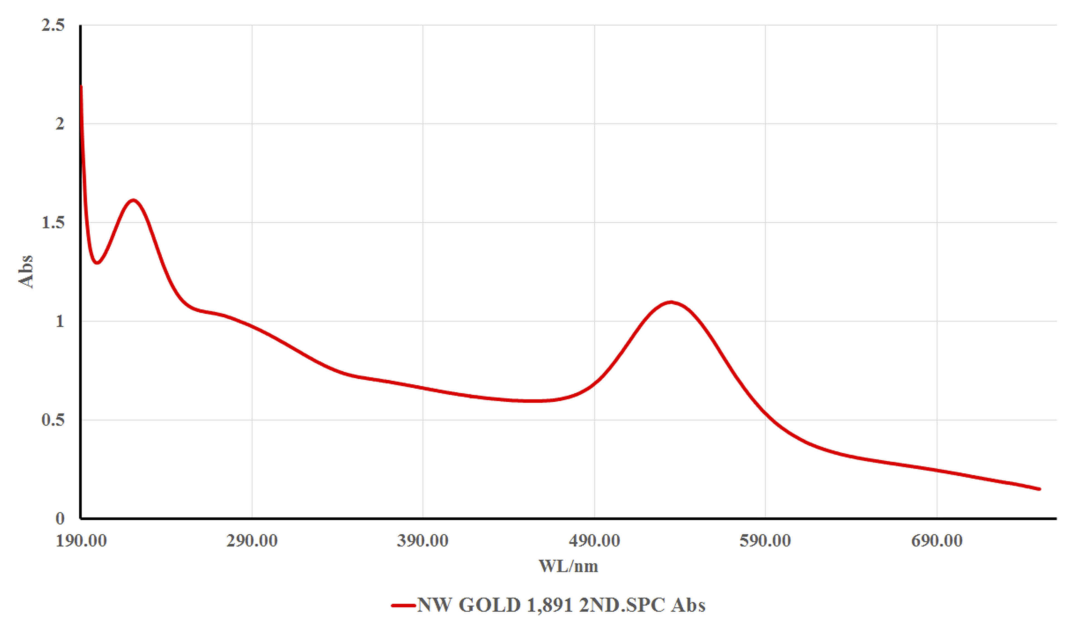

Figure I UV-Vis absorption spectrum of gold NPs at $520 \mathrm{~nm}$. 


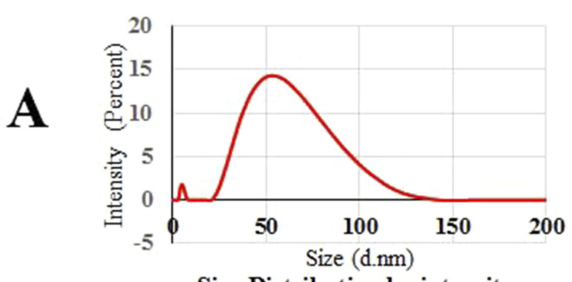

— Size Distribution by inten sity

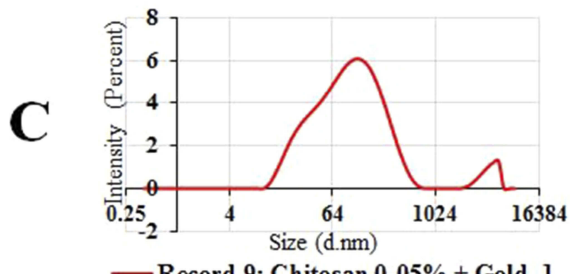

- Record 9: Chitosan 0-05\% + Gold 1
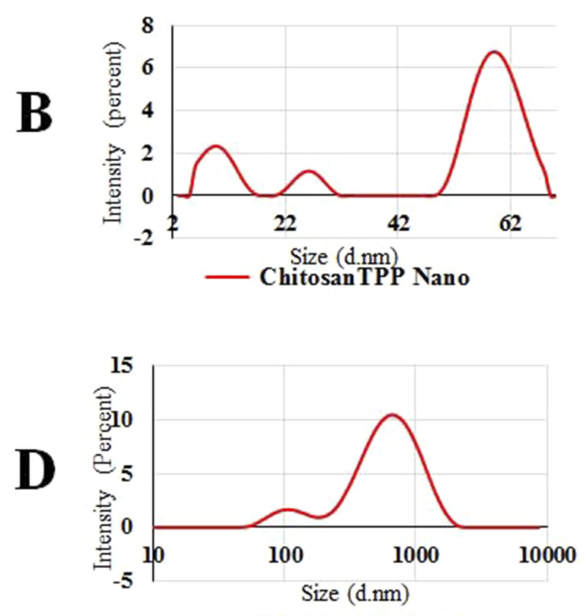

- EGCG + Chitosan

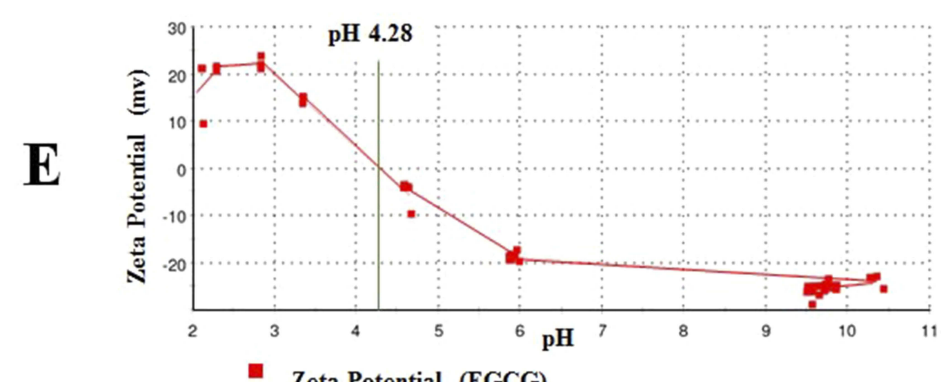

- Zeta Potential (EGCG)

Weighted Mean Zeta Potential (EGCG)

Isoelectric Point (EGCG)

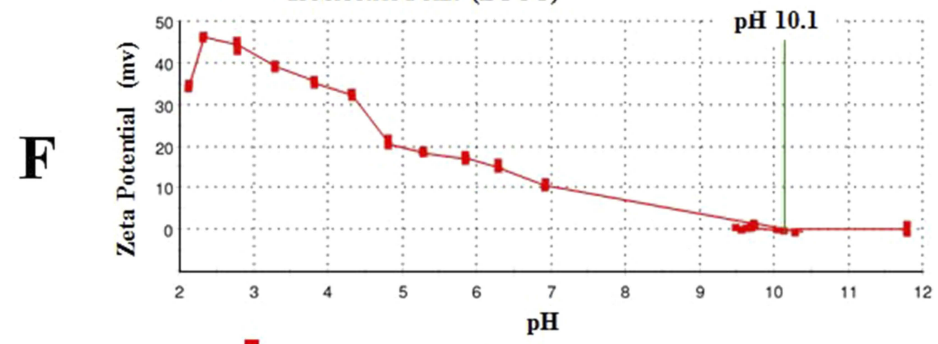

- Zeta Potential (Chitosan+Ploxamer)

Weighted Mean Zeta Potential (Chitosan+Ploxamer)

Isoelectric Point (Chitosan+Ploxamer)

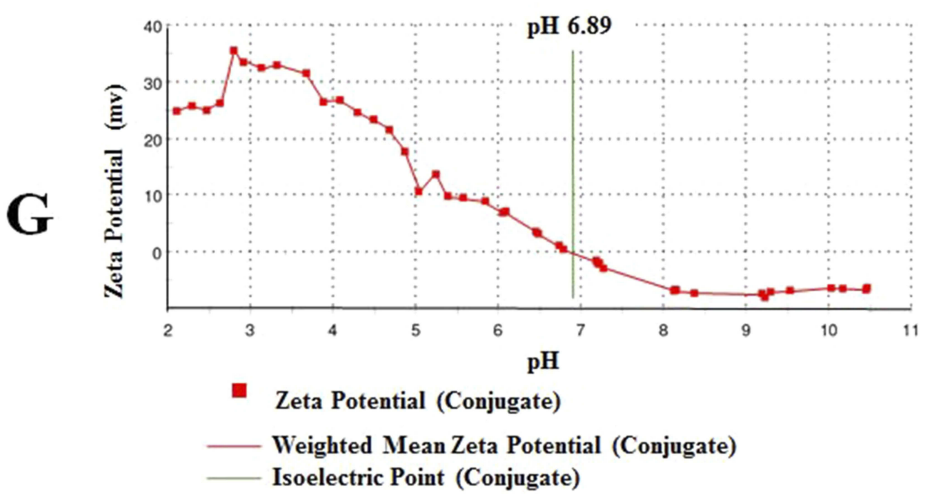

Figure 2 DLS data $(\mathbf{A})$ gold NPs (zeta size $47.03 \pm 45.55$ (d, nm) and Pdl 0.328, zeta potential $39.5 \pm 5.74$ (mV)); (B) chitosan TPP NPs (zeta size I56 \pm 45.55 (d, nm) and Pdl 0.38 I, zeta potential $-12.9 \pm 8.07(\mathrm{mV})$ ); (C) gold/chitosan NPs DLS data (zeta size 160.3 $\pm 36.17(\mathrm{~d}, \mathrm{~nm})$ and Pdl 0.58I, zeta potential 63.9 \pm 4.01 (mV); (D) EGCG/chitosan NPs (zeta size $414.8 \pm 333.8(\mathrm{~d}, \mathrm{~nm})$ and Pdl 0.363 , zeta potential $67.6 \pm 4 \mathrm{mV})$; (E) weighted mean zeta potential (EGCG), isoelectric point at pH 4.28 (EGCG), after centrifugation and auto-titration (F) weighted mean zeta potential (chitosan/poloxamer), isoelectric point at pHI0.I after centrifugation and auto-titration; (G) weighted mean zeta potential (chitosan/EGCG). Isoelectric point at $\mathrm{pH} 6.89$ (chitosan/EGCG conjugation), after centrifugation and auto-titration. 

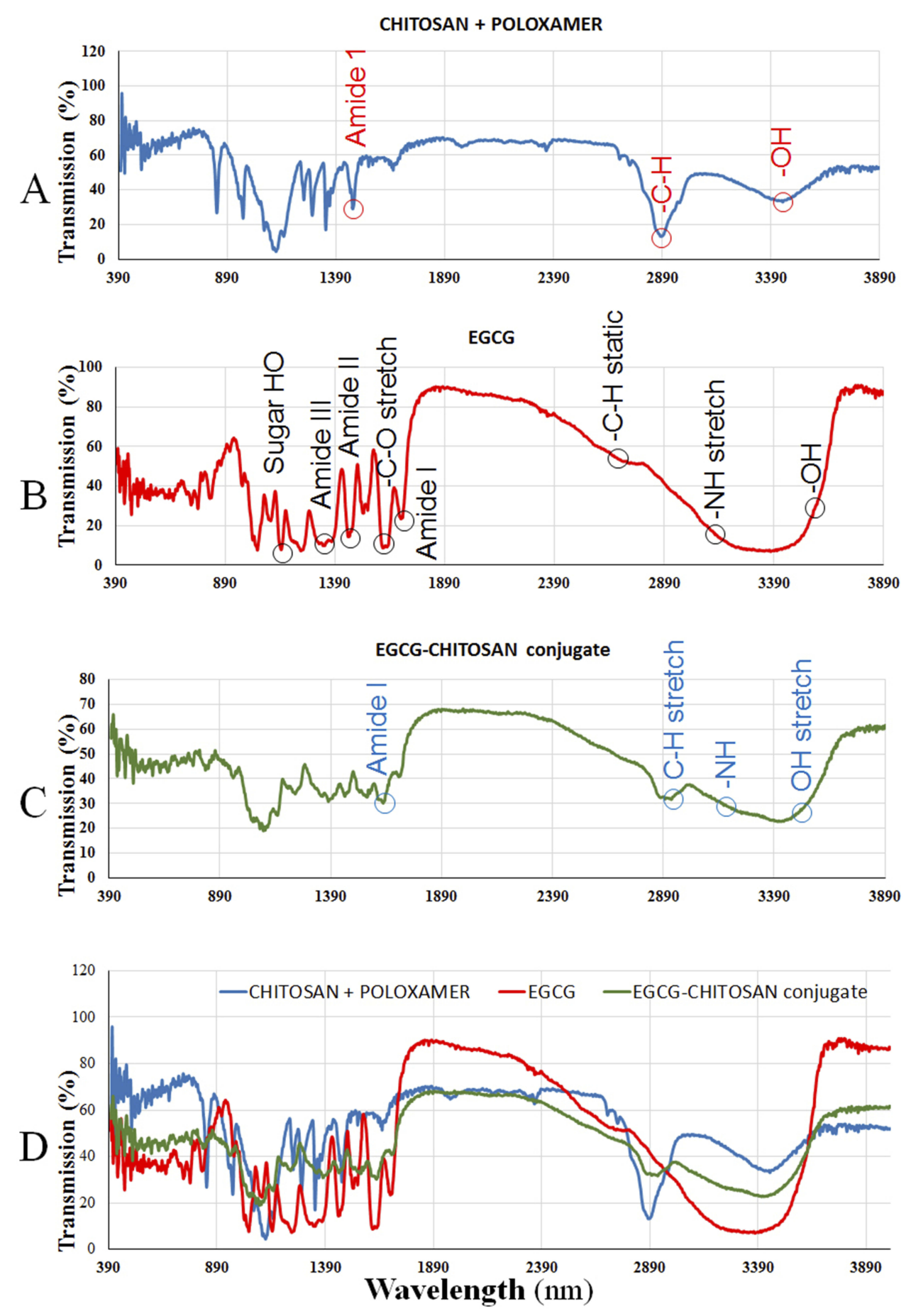

Figure 3 FTIR spectra recorded in the region of $900-4500 \mathrm{~cm}^{-1}$ of (A) chitosan/poloxamer, (B) EGCG, (C) EGCG/chitosan conjugation and self-assembled NPs and (D) combined $(\mathbf{A}-\mathbf{C})$.

FTIR Spectra And The Bindings Of EGCG-Chitosan Conjugates

FTIR data show the percentage of transmission spectral changes for chitosan, EGCG and EGCG/chitosan conjugate (shown in Figure $3 \mathrm{~A}-\mathrm{C}$ ) and with the final setting at a combined form (see Figure 3D). The infrared spectrum of chitosan/poloxamer was measured in the region of wavelength $1400-4000 \mathrm{~nm}$, while for EGCG was very strong in the regions $1000-4000 \mathrm{~nm}$, and for EGCG/chitosan conjugate was $1800-4000 \mathrm{~nm}$. However, with FTIR absorption curve shifting and intensity variations, of chitosan/poloxamer amide of I band was 


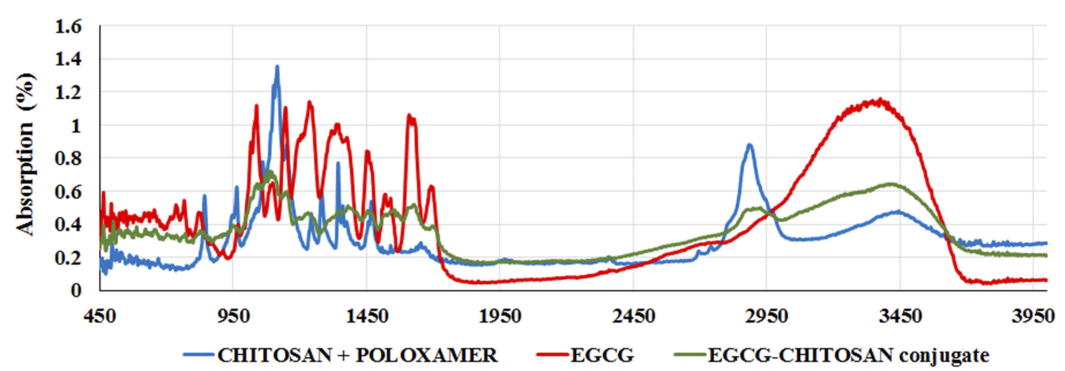

Figure 4 FTIR absorption spectra recorded in the region of $900-4500 \mathrm{~cm}^{-1}$ of (light blue) chitosan/poloxamer (red) EGCG (green) EGCG/chitosan conjugation.

approximately 500-270 $\mathrm{cm}^{-1}$, EGCG $1000-3700 \mathrm{~cm}^{-1}$ and EGCG/chitosan conjugate 1000-380 $\mathrm{cm}^{-1}$ (as reported in Figure 4), while the position of peak centers for EGCG/chitosan conjugate is clearly seen in Figure 5.

\section{X-Ray Photoelectron Spectroscopy (XPS)}

Since the objective of this study is to measure counts per second (CPS) as a function of kinetic energy (eV) of emission for electrons from atoms in the surface material, that is measured in units of $\mathrm{CPS} \mathrm{eV}$, variation in intensity for electrons with differing emission energies provides a means for characterizing the electronic state of atoms within the sample under study from which electrons are emitted and offers quantifiable information about the surface composition of EGCG/chitosan conjugate: this indicates that the conjugate was successfully formed (Figure 5).

\section{Transmission Electron Microscopy (TEM)}

Under TEM analysis, gold NPs were detected as very clear crystals, ranging in size of $47.03 \pm 45.55 \mathrm{~nm}$, with various shapes: cubes, cube-octahedrons, octahedron, tetrahedron, decahedron and icosahedron. However, the majority of the NPs were spherical in shape (see Figure 6A). Chitosan/ TPP NPs were of various sizes ranging from $156 \pm 45.55$ $\mathrm{nm}$, opaque particles (as shown in Figure 6B), with peaks clearly shown in ImageJ for gold NPs (as in Figure 6A1) and for chitosan/TPP NPs (see Figure 6B1). However, for gold/chitosan NPs, TEM shows more particle variations in size of $160.3 \pm 36.17 \mathrm{~nm}$ and shape: triangular, pentagonal and hexagonal but the majority were of spherical shape (shown in Figure 7A), with 3D peaks as seen in ImageJ (Figure 6A1). For EGCG/chitosan/TPP NPs, we observed the size of $414.8 \pm 333.8 \mathrm{~nm}$ (Figure 6B). Peaks of such variations are very clearly detected when ImageJ Software was applied (Figure 6B1).

\section{Field Emission Scanning Electron Microscopy (FESEM)}

The morphology of the four NPs: (such as gold, chitosan, gold/chitosan and EGCG/chitosan conjugation) was determined with the field emission scanning electron microscope (see Figure 7A-B1). The size of gold NPs was $47.03 \pm 45.55 \mathrm{~nm}$ (in Figure 7A). Chitosan/TPP NPs size was $156 \pm 45.55 \mathrm{~nm}$, old/chitosan was 160.3 $\pm 36.17 \mathrm{~nm}$ and EGCG/chitosan/TPP NPs were found the largest size $(414.8 \pm 333.8 \mathrm{~nm})$. The peaks were obtained by the 3D profile surface structures at different sections as $\mathrm{X}, \mathrm{Y}$ and $\mathrm{Z}$ by using Image $\mathrm{J}$ analysis: they showed NPs profile and distribution ImageJ (as shown in Figure $7 \mathrm{~A}-\mathrm{B} 1$ ).

\section{Atomic Force Microscopy (AFM)}

The 3D surface topography and section topography of gold, chitosan, gold/chitosan and EGCG/chitosan NPs were investigated over square areas of $1 \mu \mathrm{m} \times 1 \mu \mathrm{m}$ using AFM in a multimodal analysis. Topographic characterization of surface samples in amplitude and height have revealed a pattern of surface characteristics of the NPs (data not shown). Highlighted 3D surfaces for three different NPs have been estimated by the multi-fractal measures (see Figures 8 and 9, too). Topographical view of the gold NPs under the AFM confirms the small size and sharp ranging in size of $47.03 \pm 45.55 \mathrm{~nm}$, as shown in the $3 \mathrm{D}$ data image as well as the section data image (as in Figure 10A). Chitosan NPs show less crystalline nature with size average of $156 \pm 45.55 \mathrm{~nm}$ (shown in Figure 10B). Gold/chitosan-conjugated NPs appear quite larger in size 160.3 $\pm 36.17 \mathrm{~nm}$ (see Figure 10C), whereas EGCG/chitosan-conjugated NPs have shown much larger size $414.8 \pm 333.8 \mathrm{~nm}$ (reported in Figure 10D). 


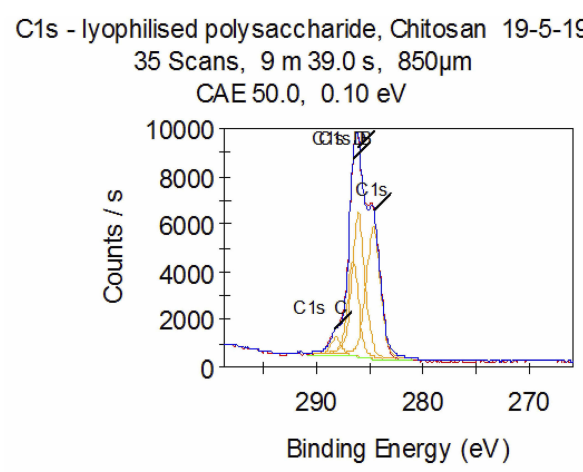

N1s - Iyophilised polysaccharide, Chitosan 19-5-19 50 Scans, $10 \mathrm{~m} 27.2 \mathrm{~s}, 850 \mu \mathrm{m}$ CAE 50.0, $0.10 \mathrm{eV}$

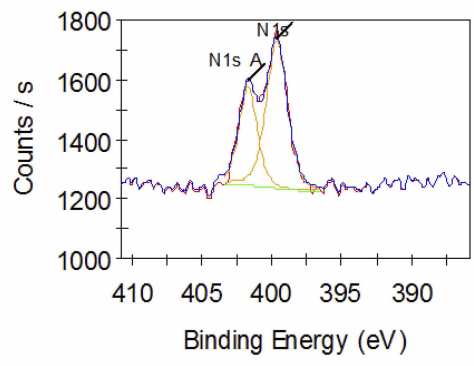

Na1s - lyophilised polysaccharide, Chitosan 19-5-19 50 Scans, $7 \mathrm{~m} 7.3 \mathrm{~s}, 850 \mu \mathrm{m}$ CAE 20.0, $0.10 \mathrm{eV}$

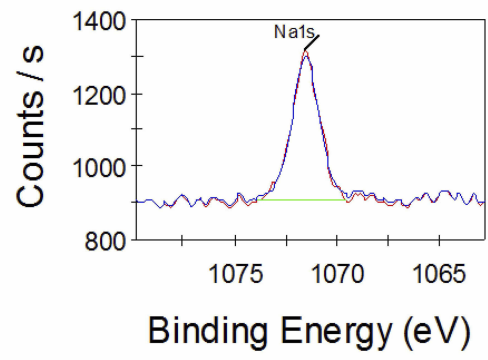

Survey - Iyophilised polysaccharide, Chitosan 19-5-1' 5 Scans, $5 \mathrm{~m} 40.1 \mathrm{~s}, 850 \mu \mathrm{m}$ CAE $150.0,1.00 \mathrm{eV}$

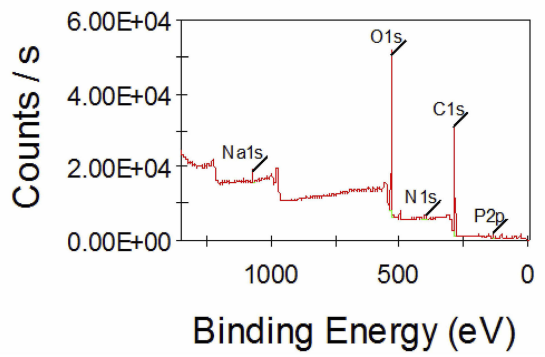

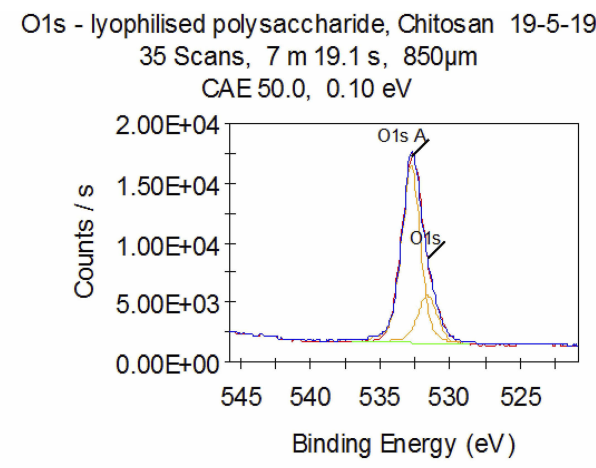

P2p - lyophilised polysaccharide, Chitosan 19-5-19 50 Scans, $8 \mathrm{~m} 22.3 \mathrm{~s}, 850 \mu \mathrm{m}$ CAE 20.0, $0.10 \mathrm{eV}$

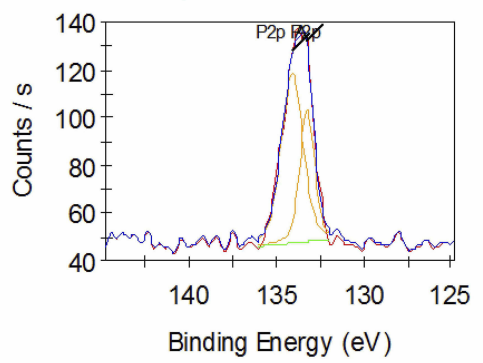

Valence - lyophilised polysaccharide, Chitosan 19-510 Scans, $1 \mathrm{~m} 53.0 \mathrm{~s}, 850 \mu \mathrm{m}$ CAE 40.0, $0.20 \mathrm{eV}$

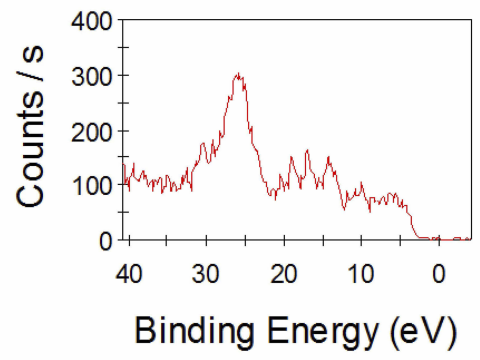

\begin{tabular}{|c|c|c|c|c|c|}
\hline Name & Peak BE & $\begin{array}{c}\text { FWHM } \\
\text { eV }\end{array}$ & $\begin{array}{c}\text { Area (P) } \\
\text { CPS.eV }\end{array}$ & Atomic \% & Q \\
\hline C1s & 284.68 & 1.49 & 10044.35 & 23.29 & 1 \\
\hline C1s B & 286.07 & 1.37 & 9906.33 & 22.98 & 1 \\
\hline C1s C & 288.21 & 1.08 & 949.83 & 2.21 & 1 \\
\hline C1s D & 286.59 & 1.21 & 5843.33 & 13.56 & 1 \\
\hline O1s A & 532.80 & 1.52 & 26957.70 & 26.05 & 1 \\
\hline O1s & 531.62 & 1.65 & 7944.73 & 7.67 & 1 \\
\hline N1s & 399.63 & 1.76 & 1034.99 & 1.23 & 1 \\
\hline N1s A & 401.71 & 1.55 & 603.71 & 0.72 & 1 \\
\hline P2p & 133.25 & 0.99 & 64.69 & 0.39 & 1 \\
\hline P2p A & 134.10 & 1.47 & 121.70 & 0.73 & 1 \\
\hline Na1s & 1071.54 & 1.55 & 709.64 & 1.18 & 1 \\
\hline
\end{tabular}

Elemental ID and Quantification

Figure 5 XPS spectra of lyophilized EGCG/chitosan conjugate and a table of elemental ID and quantification at various binding energy (eV) levels.

\section{Discussion}

The structure, size, distribution and characteristics of the four NPs prepared and studied in this work are fully in agreement with previous studies ${ }^{7,16-19}$ and further extended when their morphological characteristics have been well investigated in TEM, FESEM and 

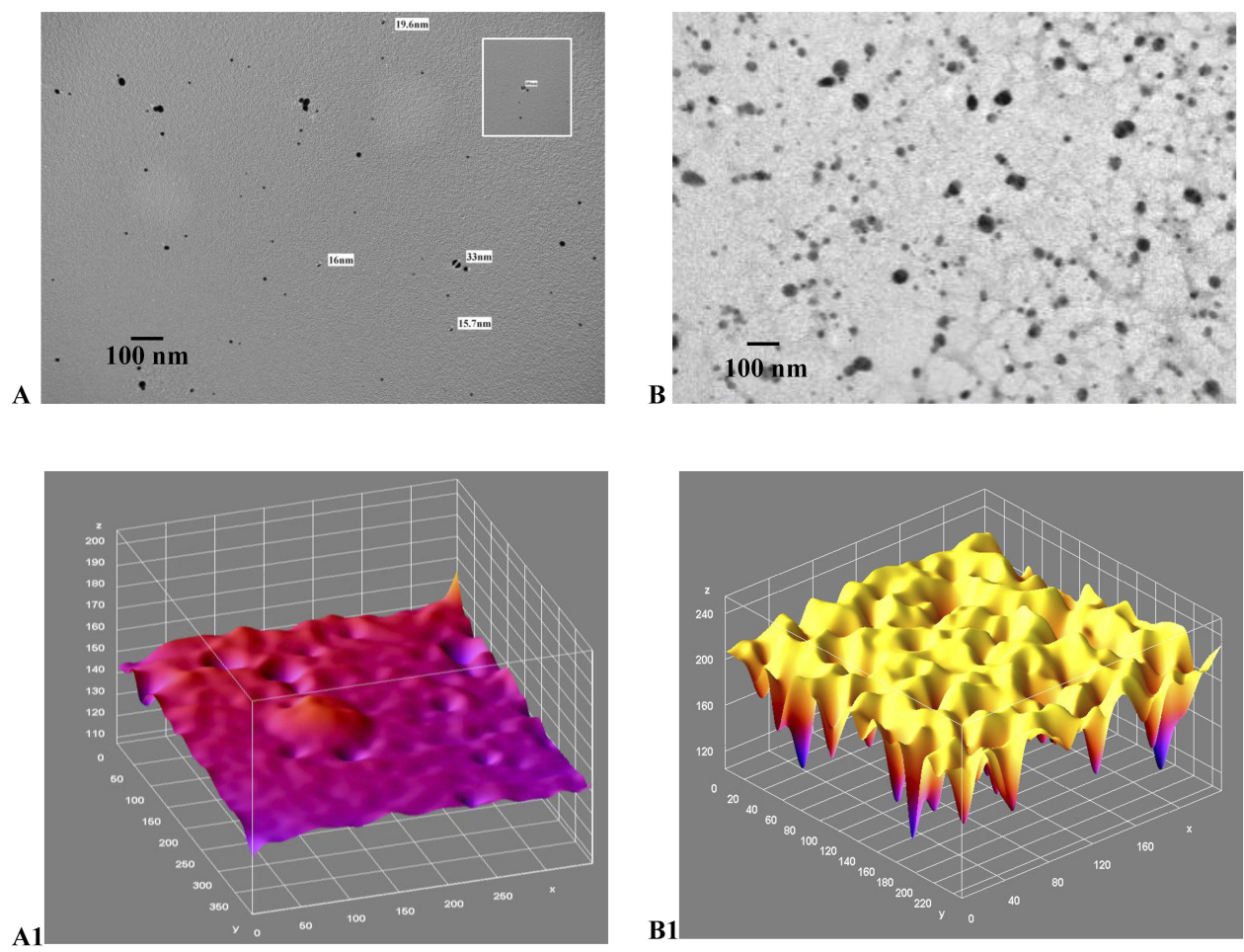

Figure 6 (A-B I): TEM image and corresponding ImageJ analysis. (A) TEM for gold NPs of various size and shape, size $47.03 \pm 45.55$ (d, nm); (B) TEM image of chitosan/TPP NPs, size 156 \pm 45.55 (d, nm); (AI) ImageJ for (A and BI) ImageJ for 6B with peaks denoting the intensity of the NPs in each case. Scale bars $300 \mathrm{~nm}$.
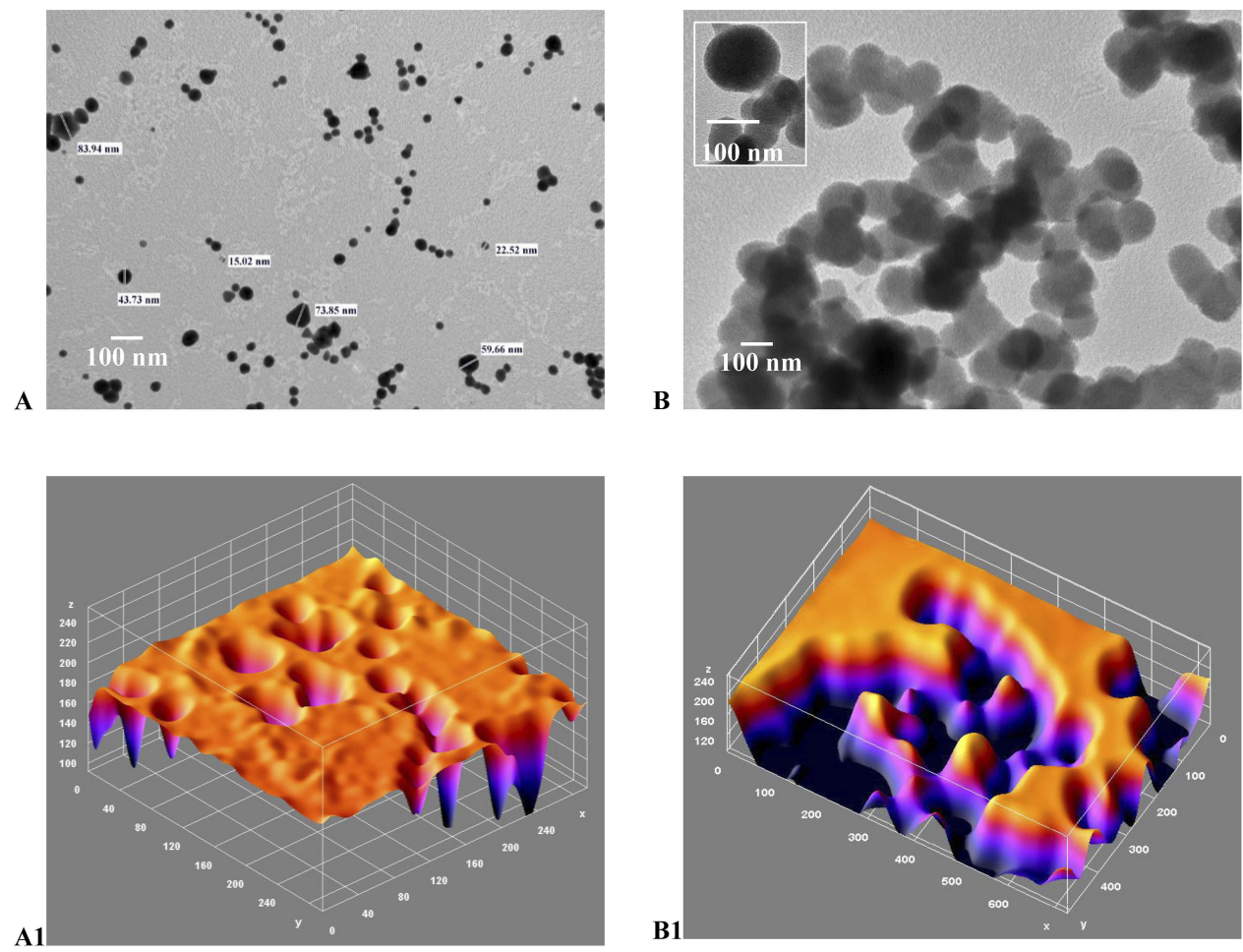

Figure 7 (A-BI): TEM image and corresponding Imagej analysis. (A) TEM for gold/chitosan NPs of various size and shape, size $160.3 \pm 36.17$ (d, nm); (B) TEM image of EGCG/chitosan/TPP NPs, size 4I4.8 \pm 333.8 (d, nm); (AI) ImageJ for (A and B I) Image for 7B with peaks denoting the intensity of the NPs in each case. Scale bars $300 \mathrm{~nm}$.

AFM. $^{11,20-26}$ It has to be noted that the purpose of using gold NPs in this report was for the sake of morphological comparison with the other three NPs under study. 

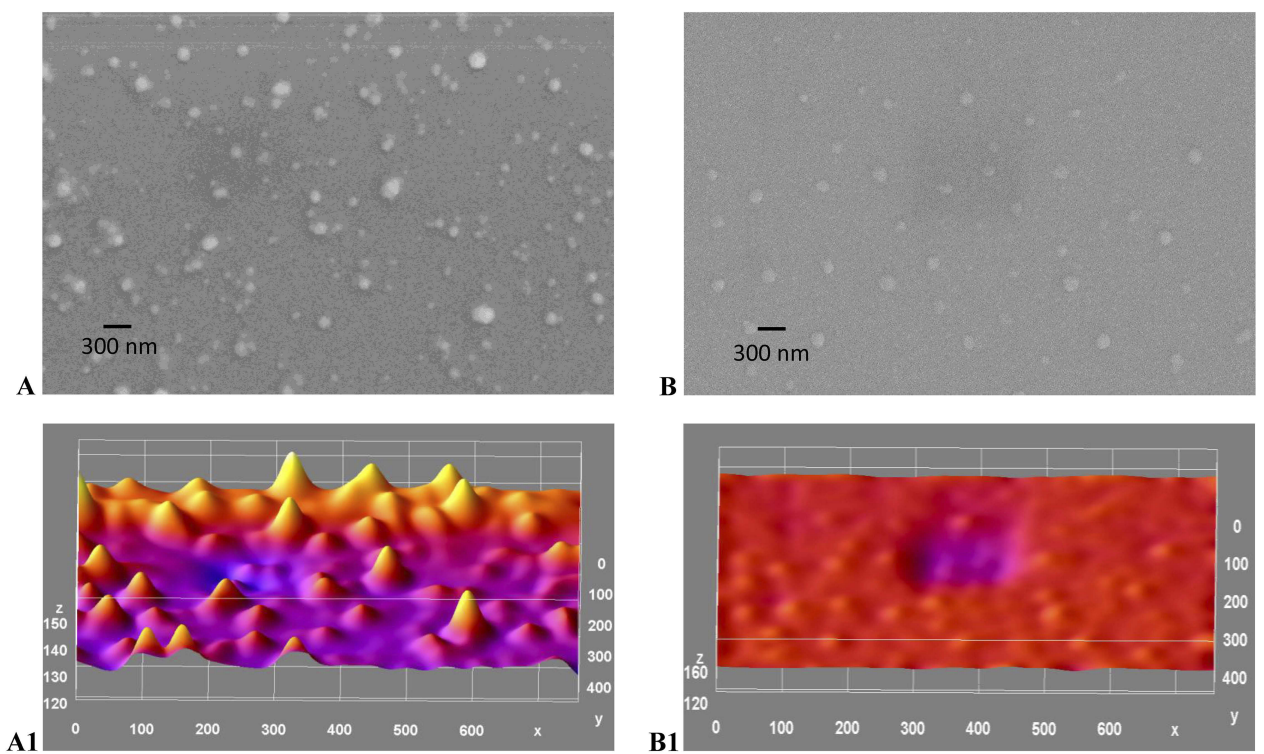

Figure 8 (A-B I): SEM image and corresponding Imagej analysis. (A) Gold NPs of various size and shape, zeta size $47.03 \pm 45.55$ (d, nm); (B) SEM image for chitosan/TPP NPs, zeta size $156 \pm 45.55$ (d, nm); (AI) Imagej for 8A and (BI) Image) for $\mathbf{A}$ with peaks denoting the intensity of the NPs in each case. Scale bars $300 \mathrm{~nm}$.

A

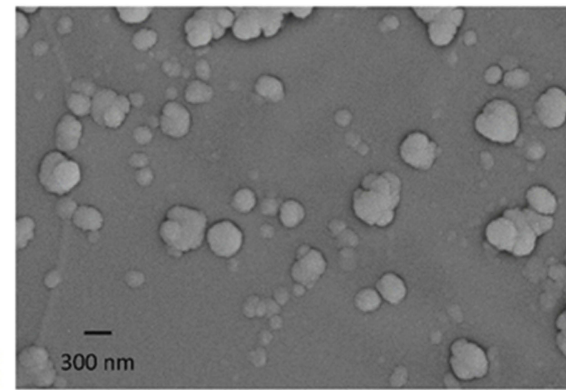

Al

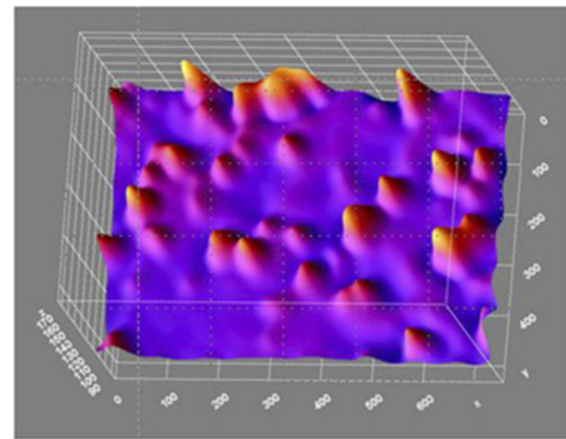

B
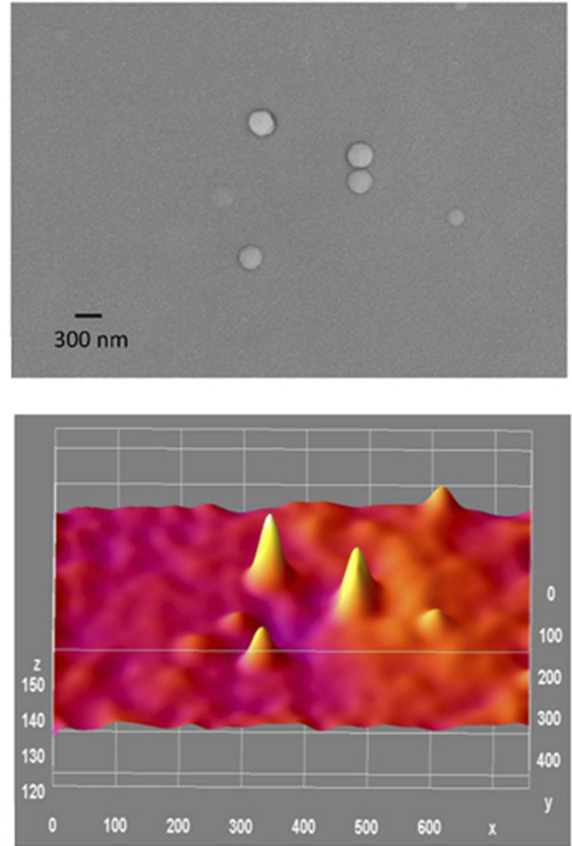

Figure 9 (A-B I): SEM image and ImageJ analysis. (A) Gold/chitosan NPs, size and shape, zeta size I60.3 \pm 36.17 (d, nm); (B) SEM image of EGCG/chitosan/TPP NPs with size of zeta size $414.8 \pm 333.8(\mathrm{~d}, \mathrm{~nm})$; (AI)Image for (A and B I) Image for (B) with peaks denoting the intensity of the NPs in each case. Scale bars $300 \mathrm{~nm}$.

\section{Drug-Loaded NPs}

The scope of this work was to optimise the ideal way to prepare chitosan (CS) as a carrier for the herbal drug EGCG NPs through the ionic interaction of a positively charged CS solution ${ }^{18,27-32}$ and negatively charged TPP

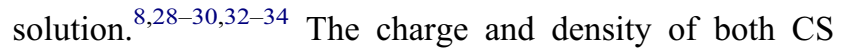
and TPP solution have a great effect on the ionic interaction. An automatic isoelectric titration instrument was used to investigate the relationship between the $\mathrm{pH}$ value and the charge density (zeta potential) of CS and TPP solution. ${ }^{4,28}$ The selection of optimum charge density is a critical step for effective ionic interactions. Apart from the individual NPs, of gold, CS, gold/CS tested, the zeta potential of CS and EGCG conjugated solution was $\mathrm{pH}$ 
A

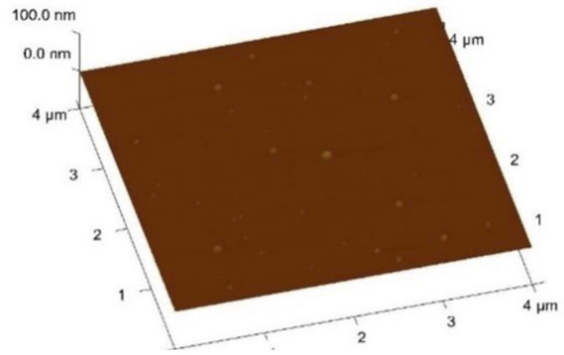

B

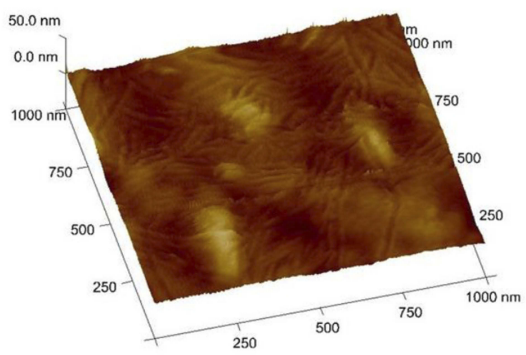

C

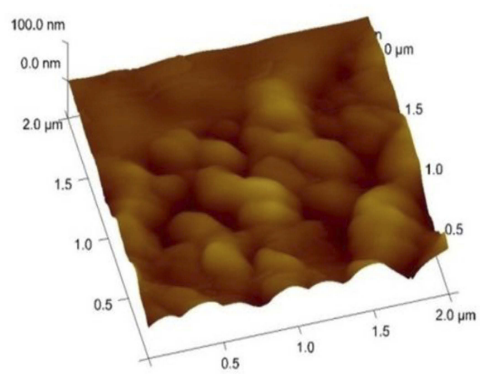

D

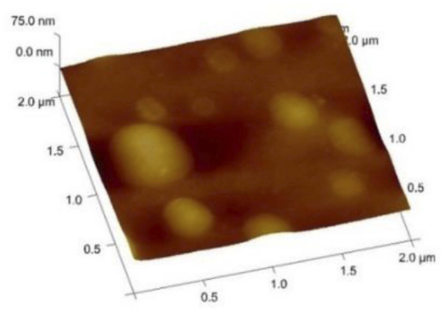

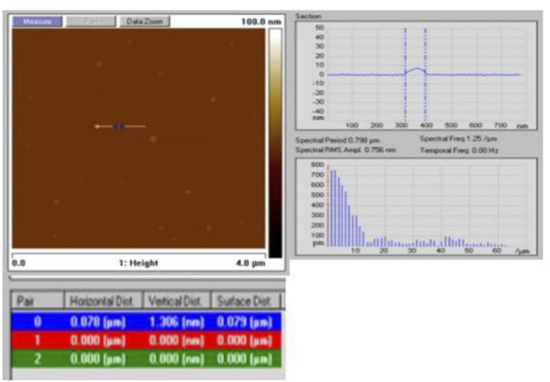
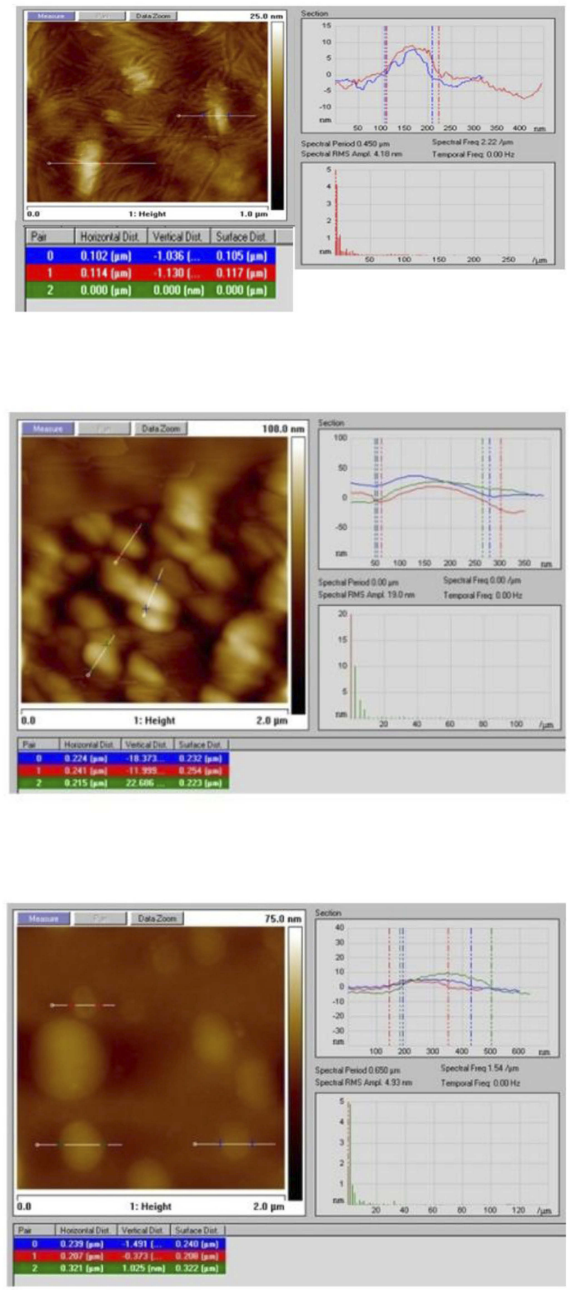

Figure 10 AFM image analysis for NPs showing 3D (left) and section data (right) of: (A) gold; (B) chitosan; (C) gold/chitosan and (D) EGCG/chitosan.

7.61 at which the zeta potential reached almost $67.6 \pm 4 \mathrm{mV}$, zeta size $414.8 \pm 333.8(\mathrm{~d}, \mathrm{~nm})$ and PdI 0.363 , zeta potential. ${ }^{6,8}$ In the case of TPP solution, the $\mathrm{pH}$ value drops dramatically from -2 to $-22.5 \mathrm{mV}$ when its $\mathrm{pH}$ increases from 5.25 to 7.25 . By considering the influence of $\mathrm{pH}$ on both CS and TPP solution, the $\mathrm{pH}$ range of 5.56.5 was chosen for the formulation of combination drug- loaded NPs in this work, because a relatively high absolute zeta potential was obtained for both TPP and CS solution in this range, which was favoured by the crosslinking of the oppositely charged solution.

The conjugation of the EGCG/CS and TPP NPs are very stable by all means. Likewise the NPs size either as individual preparation or as final conjugated EGCG/ 


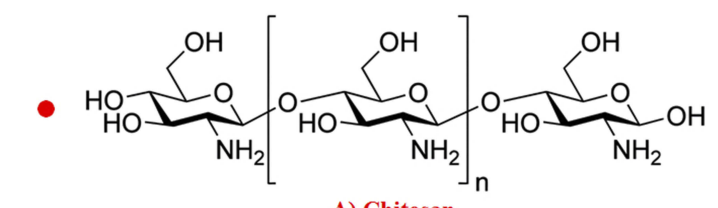

A) Chitosan

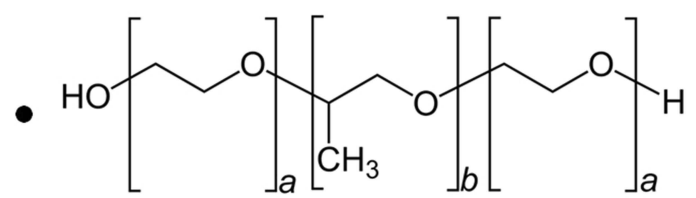

B) Poloxamer 188
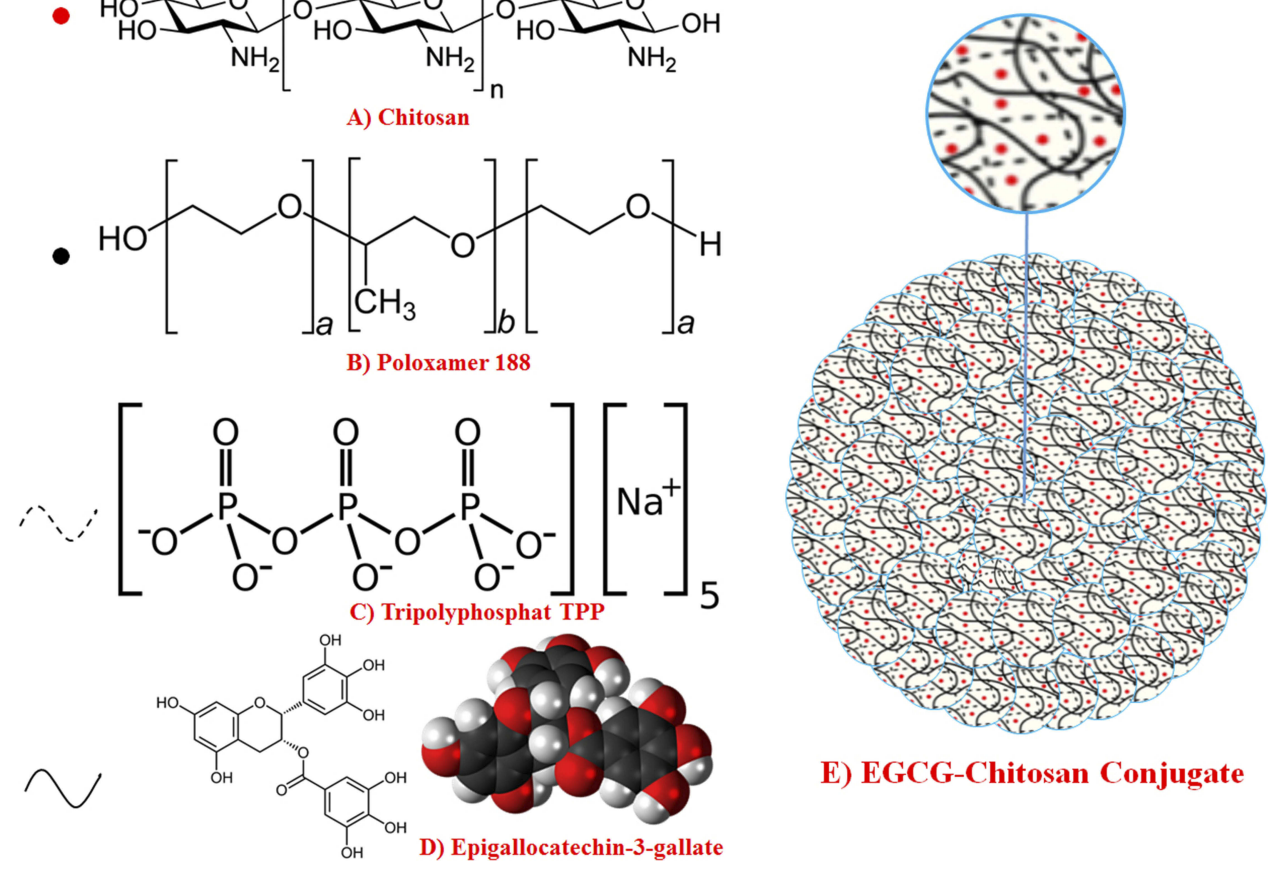

E) EGCG-Chitosan Conjugate

Figure II (A) Chemical structure of chitosan, (B) tripolyphosphate (TPP), (C) Poloxamer I88 (D) EGCG and (E) fabrication of Poloxamer (PI88) loaded with chitosan nanoparticles via ionic gelation between the positively charged amino groups of chitosan (CS) and negatively charged tripolyphosphate (TPP) with EGCG trapped in.

CSNPs is maintained in a nanometric range. Accordingly, the conjugation appears to be moderately efficient as final products, especially the conjugation of EGCG loading into chitosan that results in a final product EGCG/CS NPs that represents a model for nano-drug delivery. ${ }^{22}$

\section{Efficiency Of Chitosan NPs As Nano-}

\section{Carrier}

Chitosan is known for its biocompatibility and as penetration enhancer owing to its electronegative property, and also act as an electrostatic stabilizer. ${ }^{13,22,27}$ Being a polysaccharide, chitosan in specific aids in the attachment of the biomolecules as well render optimum stability and subsequently help to improve the uptake of the NPs. By varying chitosan solution concentrations for the formation of EGCG/CS NPs, we have demonstrated that lower concentrations of $0.01 \% \mathrm{w} / \mathrm{v}-0.05 \% \mathrm{w} / \mathrm{v}$ resulted in aggregated particles as seen in the AFM images. Otherwise, other concentrations of each component have shown no aggregation. $^{12,16,35}$

To elucidate the cause of aggregation in such chitosan concentrations, we have measured zeta potential. This measurement allows predictions about the stability of colloidal aqueous dispersions. Usually, particle aggregation is less likely to occur for charged particles with optimum zeta potential (eg, higher than $30 \mathrm{mV}$ ) due to electrostatic repulsions.

The initial components in this study were chitosan via the $\mathrm{N}$-deacetylated product of the polysaccharide chitosan combined with Poloxamer 188 which has low toxicity, high stability capacity: the possibility of interaction with chitosan through hydrogen bonding and its excellent drug-release characteristics are explored in the polymer's use as a drug delivery vehicle for a variety of therapeutic agents. ${ }^{25}$ Thus, specific interactions between EGCG and chitosan were responsible for the self-assembly of the two NPs (see Figure 11A-D).

The FTIR experiment was used to assess the functional groups present in the three components, namely chitosan, EGCG and EGCG/CS. ${ }^{36,37}$ The FTIR spectra of the original chitosan and EGCG powders as well as the EGCG/CS assembled NPs were measured. Chitosan showed absorption bands at around $900-4500 \mathrm{~cm}^{-1}$ due to the vibration of the O$\mathrm{H}$ linkage of phenolic hydroxyl groups. ${ }^{25,38}$ EGCG displayed absorption bands at $1000 \mathrm{~cm}^{-1}$ and $3500 \mathrm{~cm}^{-1}$ that are assigned to N-H (amide II) bending, respectively. Hydrogen bonding between aliphatic and aromatic $\mathrm{O}-\mathrm{H}$ groups, respectively, on chitosan and EGCG is observed for O-H stretching since this peak shifted from $1000 \mathrm{~cm}^{-1}$ for chitosan toward 
$3500 \mathrm{~cm}^{-1}$ and became broad for values of chitosan/EGCG NPs. ${ }^{38}$ Although the peaks for EGCG/CS conjugation are clearly depicted, yet they require a thorough study to extract the exact positions of all the bonds involved to extract the exact positions of all the bonds involved (as shown in Figure 5) in the "Results" section. Nevertheless, FTIR spectra demonstrated undoubtedly the successful loading of EGCG inside chitosan NPs due to specific bonds ascribed to EGCG, envisaging the possible use of such nano-construct to encapsulate and later release active molecules or drugs.

The future work will be devoted to further study in understanding the quantification of loading, mechanism and quantification of release. Investigation of interaction of NPs with biological medium, ie, in vitro data and in vivo on animal model will focus on hepatic fibrosis. For the final state of the EGCG/chitosan conjugate testing, XPS was conducted using a Thermo Scientific ESCALAB-250Xi spectrometer. The radiation source was monochromatic of AlK operating with a power of $300 \mathrm{~W}(15 \mathrm{kV}, 20 \mathrm{~mA})$ vacuum, the analysis chamber was higher than $7 \times 10^{-9}$ mbar during all the measurements. The results indicate the successful layer-by-layer assembly of the chitosan and the formation of EGCG/chitosan conjugate after reacting the EGCG with chitosan through TPP and Poloxamer 188 crosslinking.

\section{Conclusion}

In this report, chitosan/EGCG NPs have been synthesized successfully and chitosan was efficiently loaded with EGCG. The colloidal stability, zeta size, zeta potential and isoelectric $\mathrm{pH}$ 7.61, FTIR spectra (both transmission and absorption) and full bioimaging of the NPs were systematically investigated. It was demonstrated the successful preparation of gold, chitosan NPs and conjugated gold/ chitosan NPs. Such successful loading of EGCG in chitosan envisages a potential nano-drug delivery system for the future use in the treatment of hepatic fibrosis disorder.

\section{Acknowledgments}

Authors gratefully acknowledge the Nanoscopy Science Center (NSC) technical staff and $\mathrm{Mr}$ Ayman E. El-Sharkawey, Faculty of Science, RSPU Unit and NanoLab, College of Engineering, Kuwait University for the use of the equipment. Abdel-Majeed Safer wishes to thank Kuwait University for granting him 1 year sabbatical leave for performing research in nano-drug delivery in Kuwait and in CNR NANOTEC-Istituto di Nanotecnologia, Lecce, Italy.

\section{Disclosure}

The authors report no conflicts of interest in this work.

\section{References}

1. Lin $\mathrm{YH}$, Chen ZR, Lai CH, Hsieh $\mathrm{CH}$, Feng CL. Active targeted nanoparticles for oral administration of gastric cancer therapy. Biomacromolecules. 2015;16(9):3021-3032. doi:10.1021/acs.biomac. 5b00907

2. Dahiya S, Rani R, Kumar S, Dhingra D, Dilbaghi N. Chitosan-gellan gum bipolymeric nanohydrogels - a potential nanocarrier for the delivery of epigallocatechin gallate. BioNanoScience. 2017;7 (3):508-520. doi:10.1007/s12668-017-0416-0

3. Goodrich CP, Brenner MP, Ribbeck K. Enhanced diffusion by binding to the crosslinks of a polymer gel. Nat Commun. 2018;9(1):4348. doi:10.1038/s41467-018-06851-5

4. Li P, Wang Y, Peng Z, She F, Kong L. Development of chitosan nanoparticles as drug delivery systems for 5-fluorouracil and leucovorin blends. Carbohydr Polym. 2011;85(3):698-704. doi:10.1016/j. carbpol.2011.03.045

5. Mahanta AK, Senapati S, Paliwal P, Krishnamurthy S, Hemalatha S, Maiti P. Nanoparticle-induced controlled drug delivery using chitosan-based hydrogel and scaffold: application to bone regeneration. Mol Pharmaceutics. 2019;16(1):327-338. doi:10.1021/acs. molpharmaceut.8b00995

6. Xiao B, Zhang Z, Viennois E, et al. combination therapy for ulcerative colitis: orally targeted nanoparticles prevent mucosal damage and relieve inflammation. Theranostics. 2016;6(12):2250-2266. doi:10.71 50/thno. 15710

7. Granja A, Frias I, Neves AR, Pinheiro M, Reis S. Therapeutic potential of epigallocatechin gallatenanodelivery systems. Biomed Res Int. 2017;2017:5813793. doi:10.1155/2017/5813793

8. Granja A, Pinheiro M, Reis S. Epigallocatechin gallatenanodelivery systems for cancer therapy. Nutrients. 2016;8:5. doi:10.3390/nu8050307

9. Zeng L, Yan J, Luo L, Ma M, Zhu H. Preparation and characterization of (-)-Epigallocatechin-3-gallate (EGCG)-loaded nanoparticles and their inhibitory effects on human breast cancer MCF-7 cells. Sci Rep. 2017;7:45521. doi:10.1038/srep45521

10. Yang R, Tian J, Wang D, Blanchard C, Zhou Z. Chitosan binding onto the epigallocatechin loaded ferritin nanocage enhance its transport across Caco-2 cells. Food Funct. 2018;9(4):2015-2024. doi:10. $1039 / \mathrm{c} 8$ fo00097b

11. Bhumkar DR, Joshi HM, Sastry M, Pokharkar VB. Chitosan reduced gold nanoparticles as novel carriers for transmucosal delivery of insulin. Pharm Res. 2007;24(8):1415-1426. doi:10.1007/s11095-007-9257-9

12. Cafaggi S, Leardi R, Parodi B, Caviglioli G, Russo E, Bignardi G. Preparation and evaluation of a chitosan salt-poloxamer 407 based matrix for buccal drug delivery. J Control Release. 2005;102(1):159169. doi:10.1016/j.jconrel.2004.09.019

13. Lin YH, Feng CL, Lai CH, Lin JH, Chen HY. Preparation of epigallocatechin gallate-loaded nanoparticles and characterization of their inhibitory effects on Helicobacter pylori growth in vitro and in vivo. Sci Technol Adv Mater. 2014;15(4):045006. doi:10.1088/1468-6996/ $15 / 4 / 045006$

14. Available from: http://rsb.info.nih.gov/ij. USA: ImageJ is a public domain Java Image Processing program inspired by NIH. Accessed 2008.

15. Abramoff MD, Magalhaes PJ, Ram SJ. Image processing with image. J Biophotonics Intl. 2004;11:36.

16. Peter B, Bosze S, Horvath R. Biophysical characteristics of proteins and living cells exposed to the green tea polyphenol epigallocatechin3-gallate (EGCg): review of recent advances from molecular mechanisms to nanomedicine and clinical trials. EurBiophys $J$. 2017;46(1):124. doi:10.1007/s00249-016-1141-2 
17. Hoseinpour V, Ghaemi N. Green synthesis of manganese nanoparticles: applications and future perspective-A review. J PhotochemPhotobiolB. 2018;189:234-243. doi:10.1016/j.jphotobiol.2018.10.022

18. Liang J, Li F, Fang Y, et al. Cytotoxicity and apoptotic effects of tea polyphenol-loaded chitosan nanoparticles on human hepatoma HepG2 cells. Mater Sci Eng C Mater BiolAppl. 2014;36:7-13. doi:10.1016/j.msec.2013.11.039

19. Iqbal M, Usanase G, Oulmi K, et al. Preparation of gold nanoparticles and determination of their particles size via different methods. Mater Res Bull. 2016;79:97-104. doi:10.1166/jcsb.2015.1127

20. Safer A-M. Emerging possibilities of green tea as a potential treatment regimen for hepatic fibrosis. Global J Nanomed. 2017;2:1-6.

21. Li W, Yalcin M, Lin O, Ardawi M-SM, Mousa SA. Self-assembly of green tea catechin derivatives in nanoparticles for oral lycopene delivery. J Control Release. 2017;248:117-124. doi:10.1016/j.jconrel. 2017.01.009

22. Yin Y, Cui L, Yan F, Zhang Z, Li W, Wang L. Epigallocatechin gallate based magnetic gold nanoshells nanoplatform for cancer theranostic applications. J Mater Chem B. 2017;5(3):454-463. doi:10. 1039/C6TB02408D

23. Yang R, Liu Y, Gao Y, Wang Y, Blanchard C, Zhou Z. Ferritin glycosylated by chitosan as a novel EGCG nano-carrier: structure, stability, and absorption analysis. Int $J$ Biol Macromol. 2017;105:252-261. doi:10.1016/j.ijbiomac.2017.07.040

24. Safer A-MA, Hanafy NA, Bharali DJ, Cui H, Mousa SA. Effect of green tea extract encapsulated into chitosan nanoparticles on hepatic fibrosis collagen fibers assessed by atomic force microscopy in rat hepatic fibrosis model. J Nanosci Nanotechnol. 2015;15(9):64526459. doi:10.1166/jnn.2015.10608

25. Chen YC, Yu SH, Tsai GJ, Tang DW, Mi FL, Peng YP. Novel technology for the preparation of self-assembled catechin/gelatin nanoparticles and their characterization. J Agric Food Chem. 2010;58(11):6728-6734. doi:10.1021/jf1005116

26. Dube A, Nicolazzo JA, Larson I. Chitosan nanoparticles enhance the intestinal absorption of the green tea catechins (+)-catechin and (-)-epigallocatechin gallate. Eur J Pharm Sci. 2010;41(2):219-225. doi:10.1016/j.ejps.2010.06.010

27. Fan W, Yan W, Xu Z, Ni H. Formation mechanism of monodisperse, low molecular weight chitosan nanoparticles by ionic gelation technique. Colloids Surf B Biointerfaces. 2012;90:21-27. doi:10.1016/j. colsurfb.2011.09.042
28. Hu B, Ting Y, Yang X, Tang W, Zeng X, Huang Q. Nano chemo prevention by encapsulation of (-)-epigallocatechin-3-gallate with bioactive peptides/chitosan nanoparticles for enhancement of its bioavailability. ChemCommun (camb). 2012;48(18):2421-2423. doi:10.1039/c2cc17295j

29. Liang J, Cao L, Zhang L, Wan X-C. Preparation, characterization, and in vitro antitumor activity of folate conjugated chitosan coated EGCG nanoparticles. Food Sci Biotechnol. 2014;23(2):569-575. doi:10.1007/s10068-014-0078-4

30. Liang J, Yan H, Puligundla P, Gao X, Zhou Y, Wan X. Applications of chitosan nanoparticles to enhance absorption and bioavailability of tea polyphenols: a review. Food Hydrocoll. 2017;69:286-292. doi:10.1016/j.foodhyd.2017.01.041

31. Hu B, Ting Y, Zeng X, Huang Q. Cellular uptake and cytotoxicity of chitosan-case in phosphopeptides nanocomplexes loaded with epigallocatechin gallate. CarbohydrPolym. 2012;89(2):362-370. doi:10.1016/j. carbpol.2012.03.015

32. Ramkumar Ponnuraj JK, Gopalakrishnan S, Senthilnathan K, Meganathan V, Saravanan P. Formulation and characterization of epigallocatechin gallate nanoparticles. Am J Pharm Res. 2015;5(01). doi:10.1044/1980-iajpr.150122

33. Schneider CA, Rasband WS, Eliceiri KW. NIH image to ImageJ: 25 years of image analysis. Nat Method. 2012;9(7):671-675. doi:10.1038/ nmeth.2089

34. Bhumkar DR, Joshi HM, Sastry M, Pokharkar VB. Chitosan reduced gold nanoparticles as novel carriers for transmucosal delivery of insulin. Pharm Res. 2007;24:1415-1426. doi:10.1007/s11095-007-9257-9

35. Liu F, Majeed H, Antoniou J, et al. $\mathrm{pH}$ and temperature stability of (-)-epigallocatechin-3-gallate-beta-cyclodextrin inclusion complexloaded chitosan nanoparticles. Carbohydr Polym. 2016;149:340347. doi:10.1016/j.carbpol.2016.04.100

36. Fernandes Queiroz M, Karoline R, Melo T, Sabry DA, Lanzi SG, Oliveira Rocha HA. Does the use of Chitosan contribute to oxalate kidneystone formation? Mar Drugs. 2015;13:141-158. doi:10.3390/md13010141

37. Manikandan M, Kannan K, Manavalan R. Compatibility studies of camptothecin with various pharmaceutical excipients used in the development of nanoparticle formulation. Int J Pharm Pharm Sci. 2013;5:315-321.

38. Safer A-M. A quantitative description of lipid and extracellular matrix proteinaceous fibers in hepatic fibrosis of a rat model by imagej using nano-images. J Text Sci Eng. 2017;08:03. doi:10.4172/ 2157-7439.1000446
International Journal of Nanomedicine

\section{Publish your work in this journal}

The International Journal of Nanomedicine is an international, peerreviewed journal focusing on the application of nanotechnology in diagnostics, therapeutics, and drug delivery systems throughout the biomedical field. This journal is indexed on PubMed Central, MedLine, CAS, SciSearch ${ }^{\mathbb{R}}$, Current Contents ${ }^{\mathbb{R}} /$ Clinical Medicine, $^{2}$
Journal Citation Reports/Science Edition, EMBase, Scopus and the Elsevier Bibliographic databases. The manuscript management system is completely online and includes a very quick and fair peer-review system, which is all easy to use. Visit http://www.dovepress.com/ testimonials.php to read real quotes from published authors. 